\title{
Front Matter: Volume 8641
}

, "Front Matter: Volume 8641," Proc. SPIE 8641, Light-Emitting Diodes: Materials, Devices, and Applications for Solid State Lighting XVII, 864101 (28 March 2013); doi: 10.1117/12.2021958

SPIE. Event: SPIE OPTO, 2013, San Francisco, California, United States 


\section{PROCEEDINGS OF SPIE}

\section{Light-Emitting Diodes: Materials, Devices, and Applications for Solid State Lighting XVII}

Klaus P. Streubel

Heonsu Jeon

Li-Wei Tu

Martin Strassburg

Editors

4-7 February 2013

San Francisco, California, United States

Sponsored and Published by

SPIE 
The papers included in this volume were part of the technical conference cited on the cover and title page. Papers were selected and subject to review by the editors and conference program committee. Some conference presentations may not be available for publication. The papers published in these proceedings reflect the work and thoughts of the authors and are published herein as submitted. The publisher is not responsible for the validity of the information or for any outcomes resulting from reliance thereon.

Please use the following format to cite material from this book:

Author(s), "Title of Paper," in Light-Emitting Diodes: Materials, Devices, and Applications for Solid State Lighting XVII, edited by Klaus P. Streubel, Heonsu Jeon, Li-Wei Tu, Martin Strassburg, Proceedings of SPIE Vol. 8641 (SPIE, Bellingham, WA, 2013) Article CID Number.

ISSN: 0277-786X

ISBN: 9780819494108

Published by

SPIE

P.O. Box 10, Bellingham, Washington 98227-0010 USA

Telephone +1 3606763290 (Pacific Time) · Fax +1 3606471445

SPIE.org

Copyright (@ 2013, Society of Photo-Optical Instrumentation Engineers.

Copying of material in this book for internal or personal use, or for the internal or personal use of specific clients, beyond the fair use provisions granted by the U.S. Copyright Law is authorized by SPIE subject to payment of copying fees. The Transactional Reporting Service base fee for this volume is $\$ 18.00$ per article (or portion thereof), which should be paid directly to the Copyright Clearance Center (CCC), 222 Rosewood Drive, Danvers, MA 01923. Payment may also be made electronically through CCC Online at copyright.com. Other copying for republication, resale, advertising or promotion, or any form of systematic or multiple reproduction of any material in this book is prohibited except with permission in writing from the publisher. The CCC fee code is 0277-786X/13/\$18.00.

Printed in the United States of America.

Publication of record for individual papers is online in the SPIE Digital Library.

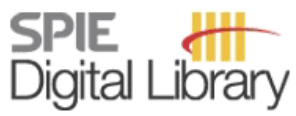

SPIEDigitalLibrary.org

Paper Numbering: Proceedings of SPIE follow an e-First publication model, with papers published first online and then in print and on CD-ROM. Papers are published as they are submitted and meet publication criteria. A unique, consistent, permanent citation identifier (CID) number is assigned to each article at the time of the first publication. Utilization of CIDs allows articles to be fully citable as soon as they are published online, and connects the same identifier to all online, print, and electronic versions of the publication. SPIE uses a six-digit CID article numbering system in which:

- The first four digits correspond to the SPIE volume number.

- The last two digits indicate publication order within the volume using a Base 36 numbering

system employing both numerals and letters. These two-number sets start with 00, 01, 02, 03, 04, $05,06,07,08,09,0 A, 0 B \ldots$. 0Z, followed by 10-1Z, 20-2Z, etc.

The CID Number appears on each page of the manuscript. The complete citation is used on the first page, and an abbreviated version on subsequent pages. Numbers in the index correspond to the last two digits of the six-digit CID Number. 


\title{
Contents
}

\author{
vii Conference Committee \\ ix Group IV photonics for the mid infrared (Plenary Paper) [8629-1] \\ R. Soref, The Univ. of Massachusetts at Boston (United States) \\ xxv Light in a twist: optical angular momentum (Plenary Paper) [8637-2] \\ M. J. Padgett, Univ. of Glasgow (United Kingdom)
}

HIGH CURRENT PERFORMANCE AND DROOP EFFECT IN LEDS I

864103 Optimizing the multiple quantum well thickness of an InGaN blue light emitting diode [8641-2]

B. XU, Tianjin Univ. (China) and Chang Gung Univ. (China); J. L. Zhao, S. G. Wang, H. T. Dai, Tianjin Univ. (China); S.-F. Yu, National Cheng Kung Univ. (Taiwan); R.-M. Lin, F.-C. Chu, C.-H. Huang, Chang Gung Univ. (Taiwan); X. W. Sun, South Univ. of Science and Technology (China)

864104 Direct green LED development in nano-patterned epitaxy (Invited Paper) [8641-3]

C. Wetzel, T. Detchprohm, Rensselaer Polytechnic Institute (United States)

\section{LED FABRICATION AND SSL I}

864107 LED color mixing with diffractive structures [8641-6]

T. Bonenberger, Hochschule Ravensburg-Weingarten (Germany) and Karlsruhe Institute of Technology (Germany); J. Baumgart, Hochschule Ravensburg-Weingarten (Germany);

S. Wendel, C. Neumann, Karlsruhe Institute of Technology (Germany)

\section{OLEDS AND OLED LIGHTING}

8641 OA Near-field photometry for organic light-emitting diodes (Invited Paper) [8641-10] R. Li, Univ. of Toronto (Canada); K. Harikumar, Lumentra Inc. (Canada); A. Isphording, Philips Technologie GmbH (Germany); V. Venkataramanan, Univ. of Toronto (Canada) and Lumentra Inc. (Canada)

$8641 \mathrm{OB}$ Numerical analysis of nanostructures for enhanced light extraction from OLEDs [8641-11] L. Zschiedrich, JCMwave GmbH (Germany); H. J. Greiner, Philips Research Aachen (Germany); S. Burger, F. Schmidt, JCMwave GmbH (Germany) and Zuse Institute Berlin (Germany) 
8641 OE Polychromatic white LED using GaN nano pyramid structure (Invited Paper) [8641-15] T. Kim, J. Kim, M. Yang, Y. Park, U.-I. Chung, Samsung Advanced Institute of Technology (Korea, Republic of); Y. Ko, Y. Cho, Korea Advanced Institute of Science and Technology (Korea, Republic of)

8641 OG Introduction of the moth-eye patterned sapphire substrate technology for cost-effective high-performance LEDs (Invited Paper) [8641-17]

K. Naniwae, M. Mori, T. Kondo, A. Suzuki, T. Kitano, EL-SEED Corp. (Japan); S. Kamiyama, EL-SEED Corp. (Japan) and Meijo Univ. (Japan); M. Iwaya, T. Takeuchi, I. Akasaki, Meijo Univ. (Japan)

$8641 \mathrm{OH} \quad \mathrm{NiO}$ as hole transport layers for all-inorganic quantum dot LEDs [8641-18]

L. Y. Tang, X. L. Zhang, H. T. Dai, Tianjin Univ. (China); J. L. Zhao, Tianjin Univ. (China) and Shanghai Juntech Co. Ltd. (China); S. G. Wang, Tianjin Univ. (China); X. W. Sun, South Univ. of Science and Technology (China)

$86410 \mathrm{~J}$ Fabrication and characterization of nitride-based blue light-emitting diodes on moth-eye patterned sapphire substrate (MPSS) [8641-20]

T. Tsuchiya, S. Umeda, M. Sowa, Meijo Univ. (Japan); T. Kondo, T. Kitano, M. Mori, A. Suzuki, K. Naniwae, EL-SEED Corp. (Japan); H. Sekine, DIC Corp. (Japan); M. Iwaya, T. Takeuchi,

S. Kamiyama, Meijo Univ. (Japan); I. Akasaki, Meijo Univ. (Japan) and Nagoya Univ. (Japan)

$86410 K \quad$ Plasmon-enhanced upconversion for $\mathrm{Yb}^{3+} / \mathrm{Er}^{3+}$ doped in $\mathrm{Y}_{2} \mathrm{O}_{3}$ [8641-21]

M. Pokhrel, J. Yang, G. A. Kumar, B. Yust, D. K. Sardar, Univ. of Texas at San Antonio (United States)

$8641 \mathrm{OL} \quad$ ZnO nanowire-based light-emitting diodes with funable emission from near-UV to blue [8641-61]

T. Pauporté, O. Lupan, Lab. d'Électrochimie, CNRS, ENSCP-Chimie-Paristech 11 (France); B. Viana, LCMCP, ENSCP-Chimie-Paristech 11 (France); T. le Bahers, Lab. d'Électrochimie, CNRS, ENSCP-Chimie-Paristech 11 (France)

NOVEL SUBSTRATES FOR LEDS

$86410 N \quad$ MOVPE-grown $n-\ln _{x} G_{a_{1-x} N}(x \sim 0.5) / p-S i(111)$ template as a novel substrate (Invited Paper) [8641-22]

A. Yamamoto, Univ. of Fukui (Japan) and JST-CREST (Japan); A. Mihara, K. Sugita, Univ. of Fukui (Japan); V. Yu. Davydov, Ioffe Physical-Technical Institute (Russian Federation);

N. Shigekawa, Osaka City Univ. (Japan)

NOVEL TECHNOLOGIES FOR LED DESIGN AND FABRICATION I

8641 OR Thermal management and light extraction in multi-chip and high-voltage LEDs by cup-shaped copper heat spreader technology (Invited Paper) [8641-26]

R.-H. Horng, H.-L. Hu, L.-S. Tang, S.-L. OU, National Chung Hsing Univ. (Taiwan) 
86417 Light extraction efficiency enhancement for InGaN quantum wells light-emitting diodes with GaN micro-domes [8641-42]

P. Zhao, L. Han, H. Zhao, Case Western Reserve Univ. (United States)

\section{LED FABRICATION AND SSL II}

864118 Growth of high indium InGaN films using a combined deposition technique and its application for long wavelength emitter (Invited Paper) [8641-43]

K.-C. Shen, M.-C. Jiang, T.-Y. Wang, R.-H. Horng, National Chung Hsing Univ. (Taiwan);

D.-S. Wuu, National Chung Hsing Univ. (Taiwan) and Da-Yeh Univ. (Taiwan)

864119 Light quality and efficiency of consumer grade solid state lighting products (Green Photonics Award Paper) [8641-44]

C. Dam-Hansen, D. D. Corell, A. Thorseth, P. B. Poulsen, Technical Univ. of Denmark (Denmark)

8641 1B Multispectral CMOS sensors with on-chip nanostructures for wavelength monitoring of LED devices [8641-46]

S. Junger, N. Verwaal, W. Tschekalinskij, N. Weber, Fraunhofer Institute for Integrated

Circuits (Germany)

\section{HIGH PERFORMANCE PHOSPHORS FOR LEDS}

8641 1D Quantum dot LED phosphors: performance and reliability improvements (Invited Paper)

[8641-48]

J. N. Kurtin, Pacific Light Technologies (United States)

$8641 \mathrm{lE}$ Color tunable green-yellow-orange-red erbium/europium codoped fluorolead germanate glass phosphor for application in LED illumination technology [8641-49]

A. S. Gouveia-Neto, W. S. Souza, R. O. Domingues, E. B. da Costa, L. A. Bueno, Univ. Federal Rural de Pernambuco (Brazil)

8641 1H Characterization and endurance study of aluminate/silicate/garnet/nitride phosphors for high-performance SSL [8641-53]

N. Trivellin, LightCube SRL (Italy) and Univ. degli Studi di Padova (Italy); M. Meneghini, M. Dal Lago, D. Barbisan, M. Ferretti, G. Meneghesso, E. Zanoni, Univ. degli Studi di Padova (Italy)

\section{NANOMATERIALS AND NANOSTRUCTURES FOR LEDS II}

8641 iN Effects of Ag doping on the ZnO nanorods inorganic/organic heterostructure LED [8641-60] X. L. Zhang, J. L. Zhao, S. G. Wang, H. T. Dai, Tianjin Univ. (China); X. W. Sun, South Univ. of Science and Technology (China) 
8641 IR Visual susceptibility analysis for solid state lighting of commercial PC-WLEDs [8641-8] C.-C. Tsai, C.-F. Yue, W.-P. Lin, J.-S. Liao, Far East Univ. (Taiwan)

8641 IT Performance enhancement of high-temperature glass-based phosphor-converted white light-emitting diodes employing $\mathrm{SiO}_{2}$ [8641-50]

C.-C. Tsai, Far East Univ. (Taiwan) and National Sun Yat-sen Univ. (Taiwan); C.-F. Yue, Far East Univ. (Taiwan); W.-C. Cheng, S.-S. Hu, National Sun Yat-sen Univ. (Taiwan); Y.-C. Hsu, National Pingtung Univ. of Science and Technology (Taiwan); J.-S. Liao, Far East Univ. (Taiwan); W.-H. Cheng, National Sun Yat-sen Univ. (Taiwan)

$86411 \mathrm{U}$ Characterization of low cost optical imaging system based on optical holography [8641-66]

M. Darwiesh, A. F. El Sherif, Military Technical College (Egypt)

$8641 \mathrm{IV}$ Use CCT and lighting distribution control algorithm for optimized energy saving with human factor lighting [8641-67]

C.-W. Lin, J.-M. Hwang, C.-M. Shih, K.-F. Hsu, Industrial Technology Research Institute of Taiwan (Taiwan)

8641 IW Miniaturization of remote phosphor LED packages [8641-68]

T.-X. Lee, M.-C. Tsai, Y.-H. Jhang, National Taiwan Univ. of Science and Technology

(Taiwan); C.-C. Chen, C.-Y. Huang, Instrument Technology Research Ctr. (Taiwan)

8641 IY Indium-zinc oxide transparent electrode for nitride-based light-emitting diodes [8641-70] S. Mizutani, S. Nakashima, M. Iwaya, T. Takeuchi, Meijo Univ. (Japan); S. Kamiyama, Meijo Univ. (Japan) and EL-SEED Corp. (Japan); I. Akasaki, Meijo Univ. (Japan); T. Kondo, F. Teramae, A. Suzuki, T. Kitano, M. Mori, EL-SEED Corp. (Japan); M. Matsubara, Idemitsu Kosan Co., Ltd. (Japan)

864120 Enhancement in external quantum efficiency of $365 \mathrm{~nm}$ vertical-type ultraviolet lightemitting diodes with embedded oxide structure [8641-72]

K.-C. Shen, M.-H. Yang, W.-Y. Lin, R.-H. Horng, National Chung Hsing Univ. (Taiwan);

D.-S. Wuu, National Chung Hsing Univ. (Taiwan) and Da-Yeh Univ. (Taiwan)

864121 High-quality quantum-dot-based full-color display technology by pulsed spray method [8641-73]

K.-J. Chen, H.-C. Chen, K.-A. Tsai, C.-C. Lin, H.-H. Tsai, S.-H. Chien, B.-S. Cheng, Y.-J. Hsu, National Chiao Tung Univ. (Taiwan); M.-H. Shih, Research Ctr. for Applied Sciences (Taiwan); H.-C. Kuo, National Chiao Tung Univ. (Taiwan)

864122 Tea quality and classification evaluation using multi-wavelength light-emitting diodes induced fluorescence spectroscopy [8641-74]

C. Feng, X. Liu, C. Yan, S. He, Zhejiang Univ. (China) and Joint Research Ctr. of Photonics (China); L. Mei, Zhejiang Univ. (China), Joint Research Ctr. of Photonics (China), and Lund Univ. (Sweden)

Author Index 


\section{Conference Committee}

Symposium Chair

David L. Andrews, University of East Anglia Norwich (United Kingdom)

Symposium Cochairs

Alexei L. Glebov, OptiGrate Corporation (United States)

Klaus P. Streubel, OSRAM GmbH (Germany)

Program Track Chair

Klaus P. Streubel, OSRAM GmbH (Germany)

Conference Chairs

Klaus P. Streubel, OSRAM AG (Germany)

Heonsu Jeon, Seoul National University (Korea, Republic of)

Li-Wei Tu, National Sun Yat-Sen University (Taiwan)

Conference CoChair

Martin Strassburg, OSRAM Opto Semiconductors GmbH (Germany)

Conference Program Committee

Gerd Bacher, Universität Duisburg-Essen (Germany)

Shoou-Jinn Chang, National Cheng Kung University (Taiwan)

Mitch M. C. Chou, National Sun Yat-Sen University (Taiwan)

Michael Heuken, AIXTRON SE (Germany)

Satoshi Kamiyama, Meijo University (Japan)

Jong Kyu Kim, Pohang University of Science and Technology (Korea, Republic of)

Markus Klein, OSRAM Opto Semiconductors GmbH (Germany)

Michael R. Krames, Soraa, Inc. (United States)

Hao-Chung Kuo, National Chiao Tung University (Taiwan)

Kei May Lau, Hong Kong University of Science and Technology

(Hong Kong, China)

Kurt J. Linden, Spire Corporation (United States)

Hans Nikol, Philips Lighting B.V. (Netherlands)

Joongseo Park, LG Electronics Inc. (Korea, Republic of)

E. Fred Schubert, Rensselaer Polytechnic Institute (United States)

Ross P. Stanley, Center Suisse d'Electronique et de Microtechnique SA (Switzerland) 
Session Chairs

1 High Current Performance and Droop Effect in LEDs I

Martin Strassburg, OSRAM Opto Semiconductors GmbH (Germany)

2 LED Fabrication and SSL I

Satoshi Kamiyama, Meijo University (Japan)

3 OLEDs and OLED Lighting

Christian Wetzel, Rensselaer Polytechnic Institute (United States)

4 Nanomaterials and Nanostructures for LEDs I

Li-Wei Tu, National Sun Yat-Sen University (Taiwan)

5 Novel Substrates for LEDs

Kurt J. Linden, Spire Corporation (United States)

6 Novel Technologies for LED Design and Fabrication I

Michael Krames, Soraa, Inc. (United States)

7 LED Materials and Characterizations

Andreas Waag, Technische University Braunschweig (Germany)

8 DUV LEDS

E. Fred Schubert, Rensselaer Polytechnic Institute (United States)

9 Novel Technologies for LED Design and Fabrication II

Michael Heuken, AIXTRON SE (Germany)

10 LED Fabrication and SSL II

Ulrich T. Schwarz, Fraunhofer-Institut für Angewandte Festkörperphysik (Germany)

11 High Performance Phosphors for LEDs

Vinod Adivarahan, Nitek, Inc. (United States)

12 High Current Performance and Droop Effect in LEDs II

Jong Kyu Kim, Pohang University of Science and Technology (Korea, Republic of)

13 Nanomaterials and Nanostructures for LEDs II

Ross P. Stanley, Center Suisse d'Electronique et de Microtechnique SA (Switzerland) 